\title{
Re: 3D Printing: A Revolutionary Advance for the Field of Urology?
}

\section{Rebecca Neu}

Technology Innovation Management Review March 2014, 19-24.

\section{EDITORIAL COMMENT}

3D bioprinting based on thermal inkjet has great potential to develop promising approaches in tissue engineering and regenerative medicine for organ replacement. With layer by layer assembly, 3D tissues with complex structures can be printed using scanned CT or MRI images. The traditional tissueengineering approach of seeding the isolated cells to the pre-formed solid and rigid scaffolds was introduced in 1993 by Langer and Vacanti. With the thermal inkjet printers, the viability of printed mammalian cells at the different cell concentrations were varying from 85-95\%. Bioprinting is flexible in that it can accommodate abroad variety of materials including organ-specific cells, blood vessels, smooth muscle and endothelial cells. With the 3D bioprinters, vascular or nevre systems can be enabled simultaneously during the organ construction with digital control. The research field of tissueengineering has seen explosive growth over the past five years where testing is stil primarily limited to animal specimens. In the literature, A. Atala and et al. demostared the power of 3D printing in thefield of urology. Especially, at the endstage of renal disease and bladder dysfunctions, tissue enginnering will be hopeful for the part of alternative treatment modality in nearfuture.

\section{Re: Robot-Assisted Renal Transplantation in the Retroperitoneum Tsai MK, Lee CY, Yang CY, Yeh CC, Hu RH, Lai HS}

Transpl Int 2014 May;27(5):452-7.

\section{EDITORIAL COMMENT}

The authors describe their first 10 cases of minimally invasive renal transplantation experience in the retroperitoneum with the aid of the da Vinci surgical system through a gas-less extra-peritoneal approach with a muscle sparing Gibson incision. The authors claim that they have utilized robotic arms for both vascular anastomosis and abdominal wall lifting which can be limiting in the obese patients. In recent years there is an increasing tendency for minimally invasive renal transplantation such as transperitoneal laparoscopic or robotic assisted renal transplantation. Those techniques still need modifications and search for a better technique is still in progress. In this study, mimicking the well-established open procedure with a smaller incision can be a better alternative, which requires confirmation in the future. 